\title{
Application of intelligent position and attitude adjustment technology in AIT of the space optical remote sensor
}

\author{
Zhifei Zhang ${ }^{1,}{ }^{*}$, Yi Ji ${ }^{2}$, Wenchun Jiao ${ }^{3}$ \\ ${ }^{1,2,3}$ Beijing Institute of Space Mechanics\&Electricity, Beijing, China
}

\begin{abstract}
In this paper, we present an intelligent position and attitude adjustment technology to solve the problem that the traditional pose adjustment scheme cannot meet the requirements of AIT, which is one of the most important parts in the manufacture of space optical remote sensor. The problem we have outlined deals largely with the study of a pioneer proposed hybrid pose adjustment strategy and a reanalysed the function of spatial pose adjustment. The strategy is realized as an intelligent position and attitude adjustment platform, and its mechanical analysis and practical statistics in AIT are carried out. The results prove to be encouraging, and it shows that the stiffness of the platform meets the requirements, the complexity of manufacturing the remote sensor is reduced, the total time of a single mission is reduced by nearly 18 times, the total man-time is reduced by 21 times, and the work efficiency is increased by 44 times. This work has resulted in a solution of the manufacturing efficiency and intelligent level of the space optical remote sensor be effectively improved.
\end{abstract}

\section{Introduction}

Spacecraft development is a very complex systems engineering, in which the AIT (assembly), Integration (Integration), Test (Test) stage is an important part of the spacecraft development process, the completion level plays an important role in the quality and reliability of spacecraft ${ }^{[1]}$. As one of the important members of the spacecraft, the optical remote sensor, which integrates light, machine, electricity and heat, is a complex hightech product integrated with many kinds of technologies [2], the position and attitude adjustment of parts and components have strict requirements. At present, the traditional way is to manually adjust the attitude of the simple tooling mechanism, and then push the wheel frame car through the position positioning. Compared with the use of hybrid automatic positioning and parallel Steward platform for attitude adjustment by NASA, ESA and other European and American space agencies, the main disadvantages of the traditional approach are:

1. Wheel frame needs a certain turning radius, which is limited in the field with large load and small space and is not conducive to the circulation between AIT processes.

2. Manual operation is dangerous, complicated, and requires high technical level of personnel. At the same time, it has high working intensity and low efficiency ${ }^{[3-}$ 4].

With "Made in China 2025", "Industry 4.0", and "advanced manufacturing partners of the United States" leading the way in space smart manufacturing technology, the intelligent automation of attitude and position adjustment in space optical remote sensor AIT is urgently needed in order to solve the problems of development risk, cost increase, efficiency and quality in manufacturing process ${ }^{[5]}$.

In this paper, the spatial pose adjustment function is analyzed, and the six-dimensional motion is reoptimized and re-combined. The novel Hybrid Pose Adjustment Strategy is composed of omni-directional motion and 3-DOF parallel mechanism. The strategy is realized as a 6-DOF intelligent position and attitude adjusting platform, and its stiffness is analyzed by finite element method. After the platform is put into AIT application, the usage of AIT is analyzed and counted. The results show that the use of the platform reduces the manufacturing complexity of the remote sensor, reduces the total time of a single mission by nearly 18 times, reduces the total man-time by 21 times, and increases the work efficiency by 44 times, the development efficiency and the intelligent automation level of the space optical remote sensor are greatly improved.

\section{Strategy of intelligent position and attitude adjustment technology}

\subsection{Analysis of Space Posture Adjustment Function}

\footnotetext{
*Corresponding author: Zhangzhifei: tedagn@163.com
} 
The motion of the six dimensions of space is defined as shown in figure 1 , where $\mathrm{X}, \mathrm{Y}$, and $\mathrm{Z}$ represent the threeway motion and belong to position adjustment. R1, R2, and $\mathrm{R} 3$ represent rotation around the $\mathrm{Z}, \mathrm{Y}$, and $\mathrm{x}$ axes (hereinafter referred to as rotation, roll, and pitch, respectively) and are classified as attitude adjustments. According to the conventional strategy, there are two schemes: the series scheme and the parallel scheme.

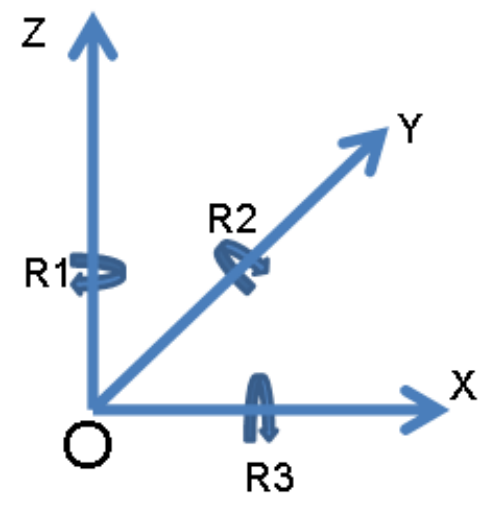

Fig. 1 Model of spatial motion

\subsubsection{Tandem scheme}

The scheme adopts six kinematic pairs in series. The kinematic pair is divided into three rotary pairs and three mobile pairs. Must be driven separately. As shown in Fig. 2(a), the scheme has mature technology, lower cost and more engineering applications.

\subsubsection{Parallel scheme}

The most typical configuration of the parallel scheme is the one proposed by the British scientist Stewart in 1965 ${ }^{[6]}$, as shown in figure 2(b). It is driven by 6 Mobile codrivers. The scheme has good stability and high control accuracy.

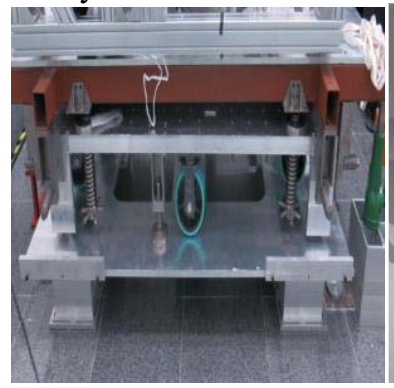

Fig. 2 (a) Tandem scheme

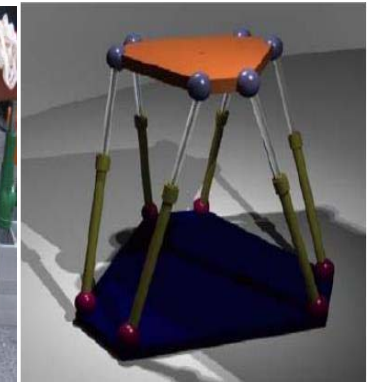

(b)Parallel scheme
Both schemes have advantages and disadvantages, as shown in Table $1^{[7-8]}$.

From Table 1, it can be seen that the conventional motion strategy cannot meet the requirement of Ait in complex optical remote sensor. Reconfiguring and recombining the various dimensional motions [9] in figure 1 , the position and attitude adjustment function is decomposed as shown in figure 3 . According to the change of the centre of mass of the load, the combination of $\mathrm{x}, \mathrm{Y}$ translation and rotation $\mathrm{R} 1$ is defined as in plane motion, which is realized by Omni Directional motion based on Mecanum Wheel technology.

Table 1 advantages and disadvantages of serial and parallel connection schemes

\begin{tabular}{|c|c|c|}
\hline $\begin{array}{l}\text { Evaluation } \\
\text { indices }\end{array}$ & Tandem scheme & Parallel scheme \\
\hline Motion range & Big & Small \\
\hline Rigidity & Low & High \\
\hline Load capacity & Low & High \\
\hline Structure & Simple & Complexity \\
\hline Accuracy & Low & High \\
\hline $\begin{array}{l}\text { Inter-process } \\
\text { flow }\end{array}$ & $\begin{array}{c}\text { Additional } \\
\text { transfer vehicle } \\
\text { required }\end{array}$ & $\begin{array}{l}\text { Additional } \\
\text { transfer vehicle } \\
\text { required }\end{array}$ \\
\hline
\end{tabular}

The $\mathrm{Z}$ translation, roll $\mathrm{R} 2$ and pitch $\mathrm{R} 2$ motions are defined as out-of-plane motions, which are realized by a 3-DOF Stewart structure using 3-RPS (revoluteprismatic-spherical) scheme. The hybrid pose adjustment strategy is composed of omni-directional motion and 3DOF parallel mechanism in series. The strategy combines the advantages of high flexibility, compact structure, high load, good expansibility of omnidirectional Planar motion, high stiffness, fast response and three-bar parallel drive of 3-RPS parallel mechanism ${ }^{[4,10]}$, it effectively solves the conflict between the AIT position and attitude adjustment requirement and the working procedure of optical remote sensor.

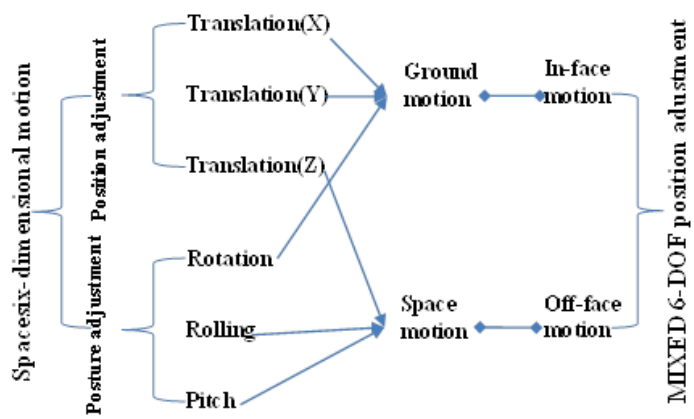

Fig. 3 Analysis of pose adjustment function and strategy configuration

\subsection{Fundamentals of Omnidirectional motion}

The general four-wheel structure with Omnidirectional motion, the more reasonable layout of the wheel set is rectangle, which can make the motion stable, the support structure stable and the safety redundancy high ${ }^{[11]}$. The principle diagram of omni-directional motion is shown in Fig. 4. By adjusting the rotational speed and steering of one to four wheels, the omni-directional motion of straight, lateral, oblique and rotary motion can be 
combined ${ }^{[12]} . \omega$ is the axial friction of the stick; and the angular velocity of the wheels.
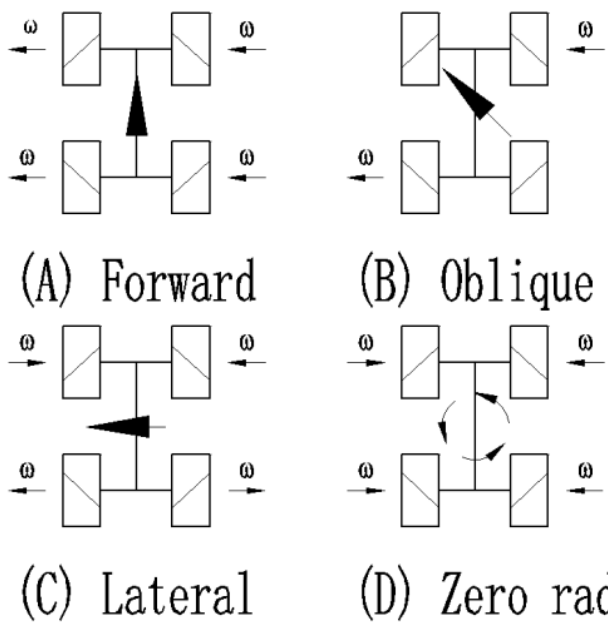

(D) Zero radius

\section{rotation}

Fig. 4 Basic principles of omni-directional motion

\subsection{Mecanum Wheel}

Mecanum Wheel is designed by the Swedish company Mecanum AB. It consists of a main hub and a rotating stick with a specific contour curve. The stick can rotate freely and its axis and the axis of the hub are distributed along the circumference of the hub at an angle of $45^{\circ}$. The outer envelope of all sticks is approximately a circle, and the equation of the Meridian curve of the Roller Profile ${ }^{[13]}$ is:

$$
m_{R} \cdots\left\{\begin{array}{c}
x^{*}(u)=d \frac{\cos ^{2} \delta}{\sin \delta} \tan u+r \sin \delta \sin u \\
z^{*}(u)=-(r \cos u-d) \sqrt{\cos ^{2} \delta \tan ^{2} u+1}
\end{array}\right.
$$

According to the structural form, Mecanum Wheel can be divided into two types: Middle Type and two-end type, each has its advantages and disadvantages: in the middle type, Mecanum Wheel bearing capacity is proportional to the thickness of the hub supporting Rod Blade, and the continuity of the roller theory envelope is inversely proportional; There is no such conflict between the two terminal forms, but they do not dominate sexually ${ }^{[14]}$. In the camera AIT stage, the site is relatively flat, considering the compatibility of heavy load, the comprehensive consideration of the selection of both ends.

\subsection{3-DOF parallel mechanism}

The 3-DOF parallel mechanism adopts a 3-RPS (revolute-prismatic-spherical) Stewart structure. Threedegree-of-freedom (DOF) parallel attitude adjusting mechanism is composed of three identical topological branches to adjust the attitude of up-down, pitch and roll. Each branch consists of rotary pairs (spherical joint) and prismatic pairs (prismatic pair) with a cylindrical pair as the driving pair. The cylinder pair is realized by threestage hydraulic cylinder to meet the requirements of large load-bearing and large yield-to-span ratio. In the initial state, the bottom bracket is in a closed state. With the motor driving, the hydraulic cylinder is lifted, which drives the bracket to push up the upper platform. After the structural adjustment is in place, four auxiliary supports are adopted to improve the stability of the lifting platform, and the top lead screw of the auxiliary supports has a larger telescopic stroke, which can meet the support requirements of the attitude adjusting device at any height and attitude angle.

\section{Equations and mathematics Realization of intelligent position and attitude adjustment platform and application effect of AIT}

The intelligent 6-DOF position and attitude adjusting platform is realized by adjusting strategy and its mechanical analysis is carried out.

\subsection{Stress Analysis of frame}

The stress analysis of the main load-bearing parts of the 6-DOF testing platform is as follows: the rated load is $2000 \mathrm{~kg}$, the safety factor is calculated and analyzed according to $3000 \mathrm{~kg}$, and the frame is analyzed under two working conditions according to the actual use.

Working Conditions 1: The platform is in a horizontal state

Boundary conditions:

Constraints: fixed constraints are imposed on the wheel assembly face;

Loading: $30000 \mathrm{~N}$ vertical downward force on the three outriggers, $1 \mathrm{~g}$ gravitational acceleration;

2) Simulation results are as shown in Fig. 5:

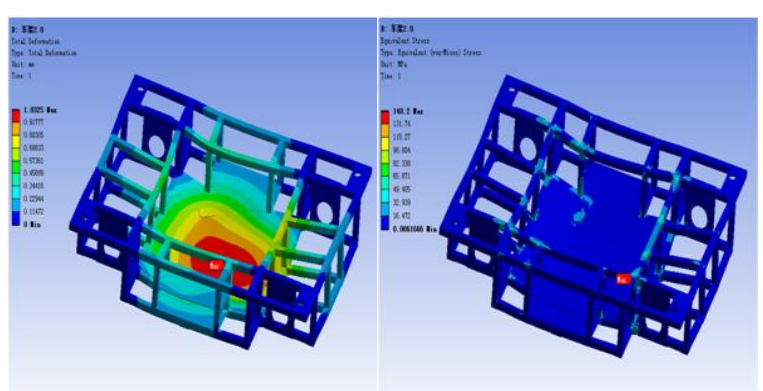

(a)Strain Nephogram $\quad$ (b) Stress Nephogram

Fig. 5 Simulation results of working condition 1

As shown in Fig. 5, the maximum deformation of the structure is $1.03 \mathrm{~mm}$, which is located in the center of the frame. The maximum stress of the structure is 148.2 MPA, which is located at the joint of the bracket mounting seat and the main beam of the frame, and the maximum equivalent stress is $65.87 \mathrm{MPA}$. While the yield strength of structure is $235 \mathrm{MPA}$, it meets the structural strength requirement of factor of safety. Therefore, the frame structure can meet the requirements of strength and stiffness. 
Working Condition 2: The upper platform is in an inclined state, as the three outriggers are not balanced. The single outrigger carries $2000 \mathrm{~kg}$, the other two outriggers each bear $500 \mathrm{~kg}$

Boundary conditions

Constraints: Fixed Constraints on Wheel Assembly face;

Loading: 30000N vertical downward force on three outriggers, 1 g gravitational acceleration;

Simulation results are as shown in Fig. 6:

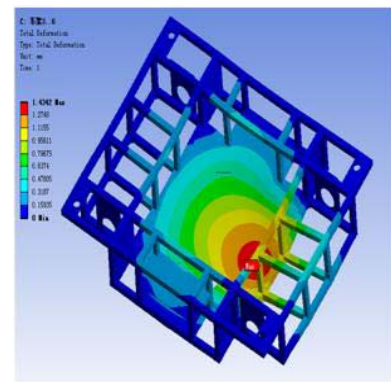

(a)Strain Nephogram

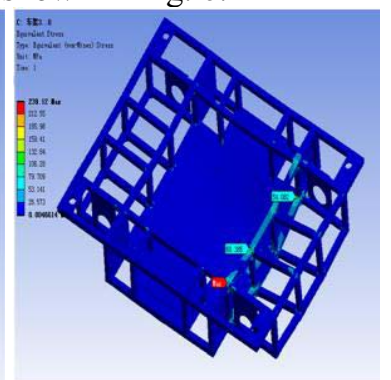

(b) Stress Nephogram
Fig. 6 Simulation results of working condition 2

As shown in Fig. 6, the maximum deformation of the structure is $1.43 \mathrm{~mm}$, which is located in the center of the frame, the maximum stress is 239.12 MPA, which is located at the joint of the outrigger mounting seat and the main beam of the frame and the maximum equivalent stress is $106.28 \mathrm{MP}$. While the yield strength of structure is $235 \mathrm{MPA}$, it meets the structural strength requirement of factor of safety. Therefore, the frame structure can meet the requirements of strength and stiffness.

In summary, the frame can meet the actual use of strength and stiffness requirements. The 6-DOF intelligent attitude control platform is shown in figure 7.

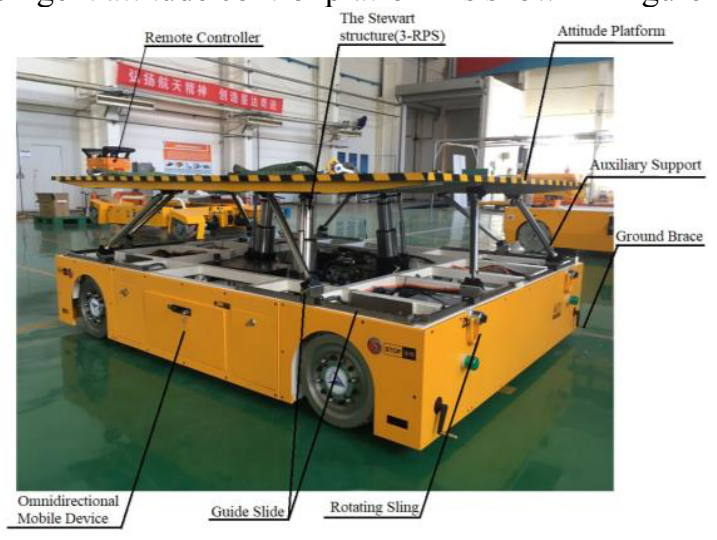

Fig. 7 The intelligent position and attitude adjustment platform

\subsection{Application Effects}

After 6-DOF intelligent attitude adjusting platform was put into the space optical remote Sensor Ait, the cost of manpower and time were effectively reduced, and the working efficiency was greatly improved. By factoring the same task before and after the use of the posture platform, as shown in figure 8, you can see a significant improvement in the performance of a single task. Before the intelligent attitude adjustment platform is used, the factors that affect the task completion progress are as high as 10 , after using it, it is reduced to 3 , which greatly reduces the complexity of the manufacturing process and the technical level of the operators, and reduces the dependence on the personnel, it improves the intelligent automation of the manufacturing process. From figure 8 , you can also see that the use of pre-factor accounted for significantly more time than after use. For each factor, time consumption ratio summary Statistics, as shown in figure 9. After the use of the platform, the total time spent per session was reduced by nearly 18 times, the total time spent per session was reduced by 21 times, and the work efficiency was increased by 44 times.

\section{Conclusion}

In view of the traditional attitude adjustment scheme cannot meet the requirements of the space optical remote Sensor in AIT, this paper analyzes the attitude adjustment function of space, and re-optimizes and recombines the six-dimensional motion, the novel Hybrid Pose Adjustment Strategy is composed of omnidirectional motion and 3-DOF parallel mechanism. In order to verify the stiffness of the combined 6-DOF attitude control platform, its model is analyzed by finite element method. After the intelligent attitude adjustment platform is put into AIT application, the usage of the platform is analyzed and counted. The results show that the use of the platform has reduced the complexity of the mission, reduced the total time by nearly 18 times, reduced the total man-time by 21 times, increased the work efficiency by 44 times, and greatly improved the development efficiency of the space optical remote sensor, and the level of intelligent automation of manufacturing, as showed in Fig.8and Fig.9.

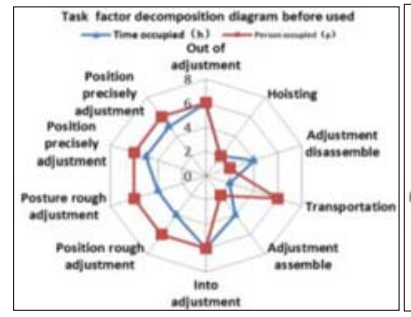

(a)Before

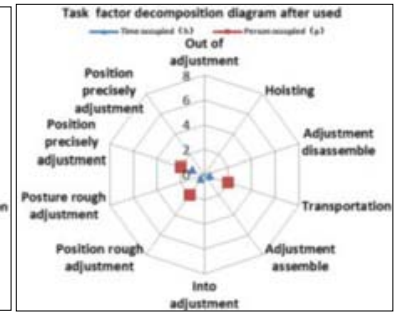

(b) After
Fig. 8 The factor decomposition before used and after used

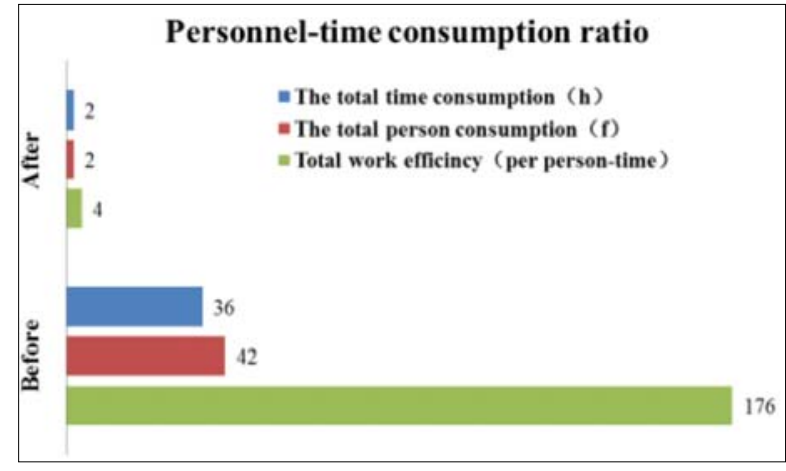

Fig. 9 The change of Personnel-time consumption ratio before used and after used 


\section{References}

1. L. Feng, D. Wu, Journal of Safety Science and Technology 3 ,102-107(2017)

2. J. Han,Technical Exchange 2(1), 58-60 (2011)

3. R. Liu, Dissertation for Master degree (Harbin, HIT,1-11,2018)

4. D. Zou, X.Ma, Dual Use Technologies \&Products, 111. (2012(2))

5. J. Sun, J. Liu, C. Zhao, SPACECRAFT ENVIRONMENT ENGINEERING 32, $577-578$ (2015)

6. M. Mustafa, Control Theory Appl 149, 156-162 (2002)

7. H. Zu, Dissertation for Master degree (Harbin, HIT, 10-12,2018)

8. S. Mao, Dissertation for Master degree (Nanjing, NUAA, 9-10,2017)

9. K. Qi, Dissertation for Master degree (Chongqing, Chongqing University, 10-11, 2016)

10. H. Yin, Dissertation for Master degree (Shenyang, Northeastern University, 2-3, 2013)

11. Y.Wang, D.Chang, JOURNAL OF MECHANICAL ENGINEERING 45, 309-310(2009)

12. Y.Zang, G.Zhu, Modern manufacturing technology and equipment 2, 14-16(2017)

13. A. Gfrerrer, Computer Aided Geometric Design 25,784-791(2008)

14. B. Hou, Y.LI, SHIP ENGINEERING 33, 84$85(2011)$ 